\title{
Pyridazine derivatives and its related compounds. Part 31. Synthesis of some disperse dyes derived from 3-amino-1H-pyrzolo[3,4-c]pyridazine and their color assessment on polyester fabrics
}

\author{
Ali AbdelHamid Deeb a,*, Mamdoh Bahgat El-Hossami b and Ahmed Awad AbdelGawad c,d \\ a Department of Chemistry, Faculty of Science, Zagazig University, Zagazig, 44519, Egypt \\ b Printing, Dyeing and Finishing Department, Faculty of Applied Arts, Helwan University, Giza, 12311, Egypt \\ c Medicinal and Aromatic Plants Department, Desert Research Center, Cairo, 11753, Egypt \\ d Department of Chemistry, Faculty of Science, Jazan University, Jizan, 2097, Saudi Arabia \\ *Corresponding author at: Department of Chemistry, Faculty of Science, Zagazig University, Zagazig, 44519, Egypt.

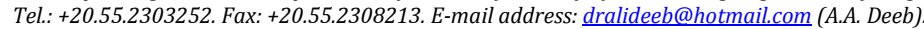

\section{ARTICLE INFORMATION}

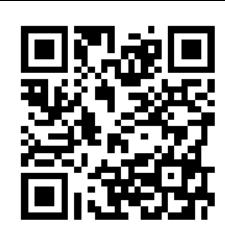

DOI: $10.5155 /$ eurjchem.5.4.639-643.1126

Received: 21 July 2014

Received in revised form: 23 August 2014

Accepted: 23 August 2014

Online: 31 December 2014

\section{KEYWORDS}

\section{Color assessment}

Dyeing properties

Fastness properties

Heterarylazo disperse dyes

Absorption spectral characteristics

3-Amino- $1 H$-pyrazolo[3,4-c]pyridazine

\section{Introduction}

Azo disperse dyes derived from heterocyclic ring systems have many advantages, such as color deepening effect as an intrinsic property of heterocyclic ring and resulting in good sublimation fastness of dyes fibers [1]. For instance, aminosubtituted pyrazole, thiazole, thiophene compounds afforded very electronegative diazo components and, consequently, provide a pronounced benzenenoid compounds [2]. We have previously reported the synthesis of novel heterocyclic systems such as 3-substituted azo-3H-pyrazolo[3,4-c]pyridazines [3], 4-[(4-arylazo-3,5-dimethylpyrazol-1-yl)carbonyl]-3,6-diphenyl pyridazine-3(2H)-one (Part 30) [4], 3-(4-arylazo-3,5-disubstituted pyrazol-1-yl)-4,5,6-triphenylpyridazines [5] and 5amino-6-[[4-arylazo-3,5-dimethylpyrazol-1-yl]-carbonyl]-3,4diphenylthieno[2,3-c]pyridazines [6] and their application to polyester fibers as disperse dyes, which gave encouraging results.

In continuation of our studies, we report here the synthesis of 6-methyl-3,4-diphenyl-7-(2-phenylhydrazono)pyrimido [1', 2':1,5]pyrazolo[3,4-c]pyridazin-8(7H)-one and 3,4-diphenyl-7(phenyldiazenyl)pyrimido[1',2':1,5]pyrazolo[3,4-c]pyridazine- 6,8-diamine derivatives use as disperse dyes for polyester. The absorption spectral characteristics, fastness properties and color assessment of the dyes are also discussed.

\section{Experimental}

\subsection{Instrumentation}

All melting points were measured using a Büchi 510 melting point apparatus and are reported uncorrected. IR spectra were recorded on a Bruker Vector 22 Germany spectrometer $(\mathrm{KBr})$. The ${ }^{1} \mathrm{H}$ NMR spectra were obtained on Varian Gemini $200 \mathrm{MHz}$ spectrometer, and chemical shifts are expressed in $\delta$ ppm using TMS as an internal standard. Mass spectra were obtained at $70 \mathrm{eV}$ using a GCMS-QP1000EX Shimadzu spectrometer. Electronic spectra were recorded on UV-visible recording Shimadzu spectrophotometer from dye solution in DMF at a concentration of $1 \times 10^{-5} \mathrm{~mole} / \mathrm{L}$.

\subsection{Synthesis}

The synthesis of 3-amino-4,5-diphenyl-1H-pyrazolo[3,4c]pyridazine 2 (Scheme 1) [7], and azobenzene compounds 


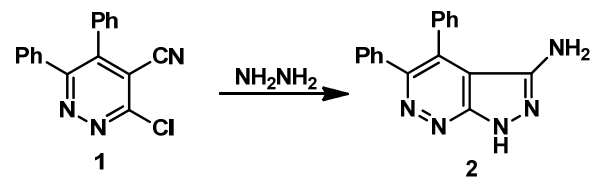

Scheme 1<smiles>CCOC(=O)NOc1ccccc1</smiles>

Scheme 2

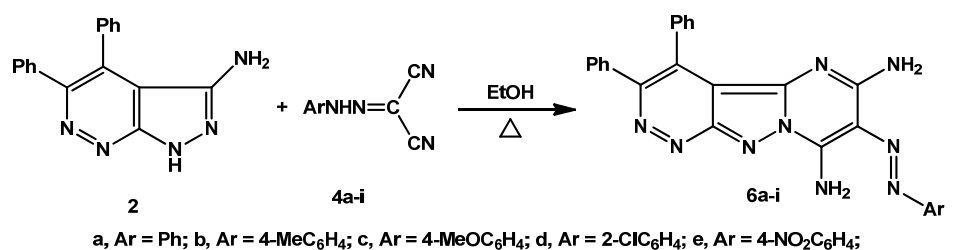

$\mathrm{f}, \mathrm{Ar}=2-\mathrm{MeC}_{6} \mathrm{H}_{4} ; \mathrm{g}, \mathrm{Ar}=3-\mathrm{MeC}_{6} \mathrm{H}_{4} ; \mathrm{h}, \mathrm{Ar}=2-\mathrm{MeOC}_{6} \mathrm{H}_{4} ; \mathrm{i}, \mathrm{Ar}=3-\mathrm{ClC}_{6} \mathrm{H}_{4}$

Scheme 3

3a-e and 4a-i [8], were conducted according to known procedures. Spectral data for compounds 3a-e and $4 \mathbf{4 a - i}$ were described in the previous parts (Scheme 2 and 3) $[9,10]$.

\subsubsection{General procedure for the synthesis of 6-methyl-3,4- diphenyl-7-(2-phenylhydrazono)pyrimido [1',2':1,5] pyrazolo[3,4-c]pyridazin-8(7H)-one (5a-e)}

To a solution of 3-amino-4,5-diphenyl-1H-pyrazolo[3,4c]pyridazine 2 (1.0 g, $3.48 \mathrm{mmoles}$ ) in a mixture of ethanol and pyridine $20 \mathrm{~mL}(3: 1, v: v)$, ethyl azobenzeneacetoacetate derivatives 3a-e (3.48 mmoles) were added, the reaction mixture was refluxed for specific reaction time. The solvent was reduced to its half and left at room temperature for $48 \mathrm{~h}$. The separated solid was filtered off, washed with ethanol (80\%), dried and recrystallized from ethanol (Scheme 2) .

6-Methyl-3,4-diphenyl-7-(2-phenylhydrazono)pyrimido [1',2': 1,5]pyrazolo[3,4-c]pyridazin-8(7H)-one (5a): Prepared from ethyl phenylazoacetoacetate 3a. Reaction time: 8 h. Color: Yellow crystals. Yield: $69 \%$. M.p.: $232-233^{\circ} \mathrm{C}$. FT-IR (KBr, v, cm1): 3446, $3176(\mathrm{NH}), 3073\left(\mathrm{CH}_{\text {arom. }}\right), 2918$ ( $\left.\mathrm{CH}_{\text {aliph. }}\right), 1671(\mathrm{C}=0)$, $1600(\mathrm{C}=\mathrm{N}), 1546(\mathrm{C}=\mathrm{C})$. UV/Vis (DMF, $\left.\lambda_{\max }, \mathrm{nm},(\log \varepsilon)\right): 267.4$ (4.1). Anal. calcd. for $\mathrm{C}_{27} \mathrm{H}_{19} \mathrm{~N}_{7} \mathrm{O}$ : C, 70.88; $\mathrm{H}, 4.19 ; \mathrm{N}, 21.43$. Found: C, 70.70; H, 4.00; N, 21.40\%.

6-Methyl-3,4-diphenyl-7-(2-(p-tolyl)hydrazono)pyrimido [1', $2 ': 1,5]$ pyrazolo[3,4-c]pyridazin-8(7H)-one $(\mathbf{5 b})$ : Prepared from ethyl 4-methylphenylazoacetoacetate $\mathbf{3 b}$. Reaction time: $10 \mathrm{~h}$. Color: Yellow crystals. Yield: 73\%. M.p.: 234-235 ${ }^{\circ} \mathrm{C}$. FT-IR (KBr, v, $\mathrm{cm}^{-1}$ ): 3454, 3157 (NH), 3077 (CHarom.), 2915, 2860 ( $\mathrm{CH}_{\text {aliph. }}$ ), $1671(\mathrm{C}=0), 1557(\mathrm{C}=\mathrm{C})$. UV/Vis (DMF, $\lambda_{\max }, \mathrm{nm},(\log \varepsilon)$ ): 266.5 (4.3). Anal. calcd. for $\mathrm{C}_{28} \mathrm{H}_{21} \mathrm{~N}_{7} \mathrm{O}$ : C, 71.32; H, 4.49; N, 20.79. Found: C, 71.20; H, 4.30; N, 20.75\%.

7-(2-(4-Methoxyphenyl)hydrazono)-6-methyl-3, 4-diphenyl pyrimido[1',2':1,5]pyrazolo[3,4-c]pyridazin-8(7H)-one (5c): Prepared from ethyl 4-methoxyphenylazoacetoacetate 3c. Reaction time: 7 h. Color: Yellow crystals. Yield: 83\%. M.p.: 248-
$249{ }^{\circ} \mathrm{C}$. FT-IR (KBr, v, cm${ }^{-1}$ ): 3441 (NH), 3056 ( $\mathrm{CH}_{\text {arom. }}$ ), 2923 $\left(\mathrm{CH}_{\text {aliph. }}\right), 2838\left(\mathrm{OCH}_{3}\right), 1597(\mathrm{C}=\mathrm{N}), 1539(\mathrm{C}=\mathrm{C}) . \mathrm{MS}(\mathrm{EI}, \mathrm{m} / z$ (\%)): $488\left(\mathrm{M}^{+}+1,3.88\right), 487\left(\mathrm{M}^{+}, 7.70\right), 353$ (0.55), 108 (100). $\mathrm{UV} / \mathrm{Vis}\left(\mathrm{DMF}, \lambda_{\max }, \mathrm{nm},(\log \varepsilon)\right.$ ): 275.7 (4.0). Anal. calcd. for $\mathrm{C}_{28} \mathrm{H}_{21} \mathrm{~N}_{7} \mathrm{O}_{2}$ : C, 68.98; H, 4.34; N, 20.11. Found: C, 68.80; H, 4.20; N, 20.10\%.

7-(2-(2-Chlorophenyl)hydrazono)-6-methyl-3, 4-diphenylpyri mido[1',2':1,5]pyrazolo[3,4-c]pyridazin-8(7H)-one (5d): Prepared from ethyl 2-chlorophenylazoacetoacetate 3d. Reaction time: 7 h. Color: Yellow crystals. Yield: 78\%. M.p.: 245-246 ${ }^{\circ} \mathrm{C}$. FT-IR ( $\left.\mathrm{KBr}, v, \mathrm{~cm}^{-1}\right): 3188(\mathrm{NH}), 3032$ ( $\left.\mathrm{CH}_{\text {arom. }}\right), 2924\left(\mathrm{CH}_{\text {aliph. }}\right)$, 1666 (C=0), 1546 (C=C), 756 (Cl-C). MS (EI, m/z (\%)):494 $\left(\mathrm{M}^{+}+2,0.57\right), 492\left(\mathrm{M}^{+}, 0.42\right), 383$ (2.89), 339 (10.58). UV/Vis (DMF, $\left.\lambda_{\max }, \mathrm{nm},(\log \varepsilon)\right): 265.2$ (4.0). Anal. calcd. for $\mathrm{C}_{27} \mathrm{H}_{18} \mathrm{ClN}_{7} \mathrm{O}: \mathrm{C}, 65.92 ; \mathrm{H}, 3.69 ; \mathrm{N}, 19.93$. Found: $\mathrm{C}, 65.90 ; \mathrm{H}$, $3.50 ; \mathrm{N}, 19.90 \%$.

6-Methyl-7-(2-(4-nitrophenyl)hydrazono)-3, 4-diphenylpyrimido[1',2':1,5]pyrazolo[3,4-c]pyridazin-8(7H)-one (5e): Prepared from ethyl 4-nitrophenylazoacetoacetate $\mathbf{3 e}$. Reaction time: 6 h. Color: Yellow crystals. Yield: $79.6 \%$. M.p.: $242-243{ }^{\circ} \mathrm{C}$. FT-IR (KBr, v, cm ${ }^{-1}$ ): 3421,3188 (NH), 3082 (CHarom.), 2931 ( $\mathrm{CH}_{\text {aliph. }}$ ), $1719(\mathrm{C}=0), 1611(\mathrm{C}=\mathrm{N}), 1554(\mathrm{C}=\mathrm{C}), 1517,1338\left(\mathrm{NO}_{2}\right)$. MS (EI, $m / z(\%)): 501\left(\mathrm{M}^{+}-1,14.7\right), 500\left(\mathrm{M}^{+}-2,50.5\right), 123(100) . \mathrm{UV} / \mathrm{Vis}$ (DMF, $\lambda_{\max }, \mathrm{nm},\left(\log \varepsilon\right.$ )): 265 (4.2). Anal. calcd. for $\mathrm{C}_{27} \mathrm{H}_{18} \mathrm{~N}_{8} \mathrm{O}_{3}$ : C, $64.54 ; \mathrm{H}, 3.61$; N, 22.30. Found: C, 64.30; H, 3.40; N, 20.30\%.

\subsubsection{General procedure for the synthesis of 3,4-diphenyl-7- (phenyldiazenyl)pyrimido[1',2':1,5]pyrazolo[3,4-c] pyridazine-6,8-diamine (6a-i)}

To a solution of 3-amino-4,5-diphenyl- $1 H$-pyrazolo[3,4c) pyridazine 2 (1.0 g, $3.48 \mathrm{mmoles}$ ) in a mixture of ethanol and pyridine $30 \mathrm{~mL}(3: 1, v: v)$, azobenzene malononitrile derivatives 4a-i (3.48 mmoles) was added, the reaction mixture was refluxed for specific time. The solvent was reduced and left at room temperature. The separated solid was filtered off, washed 
with ethanol (80\%), dried and recrystallized from ethanol (Scheme 3).

3,4-Diphenyl-7-(phenyldiazenyl)pyrimido[1',2':1,5] pyrazolo [3,4-c]pyridazine-6,8-diamine (6a): Prepared from phenylazo malononitrile 4a. Reaction time: 18 h. Color: Yellow crystals. Yield: $62.5 \%$. M.p.: $281-282{ }^{\circ} \mathrm{C}$. FT-IR (KBr, v, $\left.\mathrm{cm}^{-1}\right): 3444,3378$, 3324, 3151 ( $\mathrm{NH}_{2}$ groups), 3059 ( $\left.\mathrm{CH}_{\text {arom. }}\right), 2911\left(\mathrm{CH}_{\text {aliph. }}\right), 1613$ $(\mathrm{C}=\mathrm{N}), 1565(\mathrm{C}=\mathrm{C})$. UV/Vis $\left(\mathrm{DMF}, \lambda_{\max }, \mathrm{nm},(\log \varepsilon)\right): 269.9(4.4)$. Anal. calcd. for $\mathrm{C}_{26} \mathrm{H}_{19} \mathrm{~N}_{9}$ : C, 68.26; H, 4.19; N, 27.55. Found: C, 68.10; H, 4.00; N, 27.60\%.

3,4-Diphenyl-7-( $p$-tolyldiazenyl)pyrimido[1',2':1,5] pyrazolo [3,4-c]pyridazine-6,8-diamine (6b): Prepared from 4-methyl phenylazomalononitrile 4b. Reaction time: $24 \mathrm{~h}$. Color: Yellow crystals. Yield: $61 \%$. M.p.: $>300{ }^{\circ} \mathrm{C}$. FT-IR $\left(\mathrm{KBr}, v, \mathrm{~cm}^{-1}\right): 3467$, 3399, 3275, 3128 ( $\mathrm{NH}_{2}$ groups), 2919, $2854 \quad\left(\mathrm{CH}_{\text {aliph. }}\right)$, 1609(C=N). MS (EI, $m / z(\%)): 472\left(\mathrm{M}^{+}, 0.63\right), 119$ (2.62), 91 (5.51). UV/Vis (DMF, $\lambda_{\max }, \mathrm{nm},(\log \varepsilon)$ ): 265.4 (4.6). Anal. calcd. for $\mathrm{C}_{27} \mathrm{H}_{21} \mathrm{~N}_{9}$ : C, 68.78; H, 4.49; N, 26.74. Found: C, 68.60; $\mathrm{H}$, $4.30 ; \mathrm{N}, 26.80 \%$.

7-((4-Methoxyphenyl)diazenyl)-3,4-diphenylpyrimido $[1,2$ ': 1,5]pyrazolo[3,4-c]pyridazine-6,8-diamine (6c): Prepared from 4-methoxyphenylazomalononitrile 4c. Reaction time: $24 \mathrm{~h}$. Color: brown crystals. Yield: 70\%. M.p.: 298-299 ${ }^{\circ} \mathrm{C}$. FT-IR (KBr, $\left.v, \mathrm{~cm}^{-1}\right): 3455,3407,3262,3122\left(\mathrm{NH}_{2}\right.$ groups), $2938\left(\mathrm{CH}_{\text {aliph. }}\right)$, $2834\left(\mathrm{OCH}_{3}\right), 1598(\mathrm{C}=\mathrm{N}), 1545(\mathrm{C}=\mathrm{C}) . \mathrm{UV} / \mathrm{Vis}\left(\mathrm{DMF}, \lambda_{\max }, \mathrm{nm}\right.$, ( $\log \varepsilon$ )): 265.8 (4.1). Anal. calcd. for $\mathrm{C}_{27} \mathrm{H}_{21} \mathrm{~N}_{9} \mathrm{O}$ (487.52): C, 66.52; H, 4.34; N, 25.86. Found: C, 66.40; H, 4.20; N, 25.80\%.

7-((2-Chlorophenyl)diazenyl)-3,4-diphenylpyrimido[1',2':1,5] pyrazolo[3,4-c]pyridazine-6,8-diamine (6d): Prepared from 2chlorophenylazomalononitrile 4d. Reaction time: $14 \mathrm{~h}$. Color: yellow crystals. Yield: $81.4 \%$. M.p.: $>300^{\circ} \mathrm{C}$. FT-IR (KBr, v, cm1):3466, 3389, 3270, 3139 ( $\mathrm{NH}_{2}$ groups), 3069 (CHarom.), 2938 $\left(\mathrm{CH}_{\text {aliph }}\right), 1610$ (C=N), $1544(\mathrm{C}=\mathrm{C}), 751$ (C-Cl). MS (EI, $m / z$ (\%)):494.4 (M+ $+2,0.2), 440(100), 414$ (2.32), 380 (1.05), 337 (0.19), 352 (0.77). Anal. calcd. for $\mathrm{C}_{26} \mathrm{H}_{18} \mathrm{ClN}_{9}$ (491.94): C, 63.48; H, 3.69; N, 25.63. Found: C, 63.30; H, 3.50; N, 25.60\%.

7-((4-Nitrophenyl)diazenyl)-3,4-diphenylpyrimido[1',2':1, 5] pyrazolo[3,4-c]pyridazine-6,8-diamine (6e): Prepared from 4nitrophenylazomalononitrile 4e. Reaction time: $24 \mathrm{~h}$. Color: Red crystals. Yield: $68.2 \%$. M.p.: $>300{ }^{\circ} \mathrm{C}$. FT-IR $\left(\mathrm{KBr}, v, \mathrm{~cm}^{-1}\right)$ : 3450, 3338, $3273\left(\mathrm{NH}_{2}\right.$ groups), $1609(\mathrm{C}=\mathrm{N}), 1579(\mathrm{C}=\mathrm{C}), 1508$, $1318\left(\mathrm{NO}_{2}\right)$. MS (EI, $\left.m / z(\%)\right): 503\left(\mathrm{M}^{+}, 25.45\right), 381(3.44), 353$ (6.06), 77 (100). UV/Vis (DMF, $\lambda_{\max }, \mathrm{nm}$, $(\log \varepsilon$ )): 266 (4.5). Anal. calcd. For $\mathrm{C}_{26} \mathrm{H}_{18} \mathrm{~N}_{10} \mathrm{O} 2$ : C, 62.15; H, 3.61; N, 27.87. Found: C, 62.00; H, 3.50; N, 27.80\%.

3,4-Diphenyl-7-(o-tolyldiazenyl)pyrimido[1',2':1, 5]pyrazolo [3,4-c]pyridazine-6,8-diamine (6f): Prepared from 2-methyl phenylazomalononitrile 4f. Reaction time: $24 \mathrm{~h}$. Color: Yellow crystals. Yield: $67 \%$. M.p.: 288-289 ${ }^{\circ} \mathrm{C}$. FT-IR $\left(\mathrm{KBr}, v, \mathrm{~cm}^{-1}\right)$ : 3435, 3395, 3268, 3135 ( $\mathrm{NH}_{2}$ groups), $2915\left(\mathrm{CH}_{\text {aliph. }}\right), 1604$ $(\mathrm{C}=\mathrm{N}), 1517$ (C=C). UV/Vis (DMF, $\lambda_{\max }, \mathrm{nm}$, $(\log \varepsilon)$ ): 266.1 (4.1). Anal. calcd. for $\mathrm{C}_{27} \mathrm{H}_{21} \mathrm{~N}_{9}$ : C, 68.78; H, 4.49; N, 26.74. Found: C, 68.60; H, 4.30; N, 26.65\%.

3,4-Diphenyl-7-(m-tolyldiazenyl)pyrimido[1',2':1, 5]pyrazolo [3,4-c]pyridazine-6,8-diamine (6g): Prepared from 3-methyl phenylazomalononitrile 4g. Reaction time: 24 h. Color: Yellow crystals. Yield: $61 \%$. M.p.: $283-284{ }^{\circ} \mathrm{C}$. FT-IR (KBr, v, $\left.\mathrm{cm}^{-1}\right)$ : 3408, 3375, 3264, 3116 ( $\mathrm{NH}_{2}$ groups), 2918, $2853\left(\mathrm{CH}_{\text {aliph. }}\right)$, 1602 (C=N). MS (EI, $m / z(\%)): 396$ (0.14), 382 (0.23), 354 (0.57), 319 (0.24), 91 (100). Anal. calcd. for $\mathrm{C}_{27} \mathrm{H}_{21} \mathrm{~N}_{9}$ : C, 68.78; $\mathrm{H}, 4.49$; N, 26.74. Found: C, 68.70; H, 4.50; N, 26.70\%.

7-((2-Methoxyphenyl)diazenyl)-3,4-diphenylpyrimido [1', 2': 1,5]pyrazolo[3,4-c]pyridazine-6,8-diamine (6h): Prepared from 2-methoxyphenylazomalononitrile $\mathbf{4 h}$. Reaction time: $24 \mathrm{~h}$. Color: Orange crystals. Yield: $74.5 \%$. M.p.: $291-292{ }^{\circ}$ C. FT-IR ( $\left.\mathrm{KBr}, v, \mathrm{~cm}^{-1}\right): 3445,3389,3276,3151$ ( $\mathrm{NH}_{2}$ groups), 2912 $\left(\mathrm{CH}_{\text {aliph. }}\right), 2816\left(\mathrm{OCH}_{3}\right), 1612(\mathrm{C}=\mathrm{N}), 1538(\mathrm{C}=\mathrm{C}) . \mathrm{UV} / \mathrm{Vis}(\mathrm{DMF}$, $\left.\lambda_{\max }, \mathrm{nm},(\log \varepsilon)\right): 269.6$ (4.3). Anal. calcd. For $\mathrm{C}_{27} \mathrm{H}_{21} \mathrm{~N}_{9} \mathrm{O}: \mathrm{C}$, 66.52; H, 4.34; N, 25.86. Found: C, 66.30; H, 4.10; N, 25.75\%.
7-((3-Chlorophenyl)diazenyl)-3,4-diphenylpyrimido[1',2':1,5] pyrazolo[3,4-c]pyridazine-6,8-diamine (6i): Prepared from 3chlorophenylazomalononitrile 4i. Reaction time: $18 \mathrm{~h}$. Color: Yellow crystals. Yield: $64 \%$. M.p.: $>300{ }^{\circ} \mathrm{C}$. FT-IR $\left(\mathrm{KBr}, v, \mathrm{~cm}^{-1}\right)$ : 3433, 3333, 3269, 3161 ( $\mathrm{NH}_{2}$ groups), 3057 ( $\left.\mathrm{CH}_{\text {arom. }}\right), 2914$ ( $\mathrm{CH}_{\text {aliph.) }} 1612(\mathrm{C}=\mathrm{N}), 1561$ (C=C), 763 (C-Cl). ${ }^{1} \mathrm{H}$ NMR (400 MHz, DMSO- $\left.d_{6}, \delta, \mathrm{ppm}\right): 7.34-7.16(\mathrm{~m}, 14 \mathrm{H}, \mathrm{Ph}), 3.34(\mathrm{~s}, 4 \mathrm{H}$ $2 \mathrm{NH}_{2}$ ). UV/Vis (DMF, $\lambda_{\max }, \mathrm{nm}$, $(\log \varepsilon$ )): 266 (4.5). Anal. calcd. for $\mathrm{C}_{26} \mathrm{H}_{18} \mathrm{ClN} 9$ : C, 63.48; $\mathrm{H}, 3.69 ; \mathrm{N}, 25.63$. Found: $\mathrm{C}, 63.30 ; \mathrm{H}$, $5.60 ; \mathrm{N}, 25.55 \%$.

\subsection{High temperature dyeing method (HT)}

\subsubsection{Materials}

Scoured and bleached polyester $100 \%\left(150130 \mathrm{~g} / \mathrm{m}^{2}, 70 / 2\right.$ denier) was obtained from Misr Company for Spinning and Weaving El-Mahala El-Kobra, Egypt. The fabric was treated before dyeing with a solution containing non-ionic detergent (Sera Wash M-RK, $5 \mathrm{~g} / \mathrm{L}$ ) and sodium carbonate $(2 \mathrm{~g} / \mathrm{L}$ ) in a ratio of $50: 1$ at $60^{\circ} \mathrm{C}$ for $30 \mathrm{~min}$, and then thoroughly washed with water and air dried at room temperature.

\subsubsection{Dyeing}

The dye baths were prepared from the dye $(2 \%$ weight of fabric) to a final liquor of 50:1 (w:w). The $\mathrm{pH}$ value of the bath was adjusted to 4.5-5.0 with acetic acid (10\%) in the presence of a 1:1 ratio of the dispersing agent (Sera Gal P-LP). The temperature was raised to $1300^{\circ} \mathrm{C}$ at the rate of $7{ }^{\circ} \mathrm{C} / \mathrm{min}$, and dyeing continued for $60 \mathrm{~min}$. After dyeing, the fabrics were thoroughly washed and then subjected to a surface reduction cleaning $[(2 \mathrm{~g} \mathrm{NaOH}+2$ g sodium hydrosulphite $) / \mathrm{L}]$. The samples were heated in this solution for $30 \mathrm{~min}$ at $85{ }^{\circ} \mathrm{C}$ and then thoroughly washed and air-dried. The dyeing was performed at $2 \%$ shade by high-temperature techniques and gave generally deep and bright intense hues, ranging from yellow to orange-yellow.

\subsection{Color measurements and analyses}

\subsubsection{Color measurement}

The colorimetric parameters of the dyed polyester fabrics were determined on areflectance spectrophotometer (Table 1). The color yields of the dyed samples were determined by using the light reflectance technique performed on UV/VIS Spectrophotometer. The colorstrengths, expressed as K/S values, were determined by applying the Kubelka-Mink Equation (1).

$K / S=\left[(1-R)^{2} / 2 R\right]-\left[\left(1-R_{0}\right)^{2} / 2 R_{0}\right]$

where $R=$ Decimal fraction of the reflectance of the dyed fabric; $R_{0}=$ Decimal fraction of the reflectance of the undyed fabric; $K=$ Absorption coefficient; $S=$ Scattering coefficient.

\subsubsection{Fastness tests}

\subsubsection{Fastness to washing}

After washing using $5 \mathrm{~g} / \mathrm{L}$ of the nonionic detergent Hostapal CV and $2 \mathrm{~g} / \mathrm{L}$ of sodium carbonate at $80^{\circ} \mathrm{C}$ for $15 \mathrm{~min}$, the dyed fabrics were tested by using ISO standard methods [11]. A specimen of dyed polyester fabric was stitched between two pieces of undyed cotton and wool fabrics, all of equal length, and then washed at $95{ }^{\circ} \mathrm{C}$ for $30 \mathrm{~min}$. The staining on two pieces of undyed cotton and wool fabrics, all of equal length, and then washed at $95{ }^{\circ} \mathrm{C}$ for $30 \mathrm{~min}$. The staining on the undyed adjacent fabrics was assessed according to the International Geometric gray scale: 1-poor, 2-fair, 3-moderate, 4-good, 5- excellent. 
Table 1. Optical measurements of the synthesized monoazo disperse dyes $\mathbf{5 a}-\mathbf{a}$ and $\mathbf{6} \mathbf{a}-\mathbf{i}$ on the polyester fabricst.

\begin{tabular}{|c|c|c|c|c|c|c|c|c|c|c|}
\hline Dyes & $a^{*}$ & $b^{*}$ & $C^{*}$ & $h^{*}$ & $L^{*}$ & $\Delta E^{*}$ & $\Delta L^{*}$ & $\Delta C^{*}$ & $\Delta H^{*}$ & $\mathrm{~K} / \mathrm{S}$ \\
\hline $5 a$ & 10.36 & 71.88 & 72.62 & 81.80 & 71.03 & 00.000 & 00.000 & 00.000 & 00.000 & 19.0 \\
\hline $5 b$ & 19.20 & 82.38 & 84.59 & 76.88 & 66.16 & 14.570 & -4.869 & 11.972 & -6.727 & 24.5 \\
\hline $5 c$ & 15.52 & 85.85 & 87.24 & 79.75 & 70.22 & 14.914 & -0.806 & 14.618 & -2.842 & 21.0 \\
\hline $5 d$ & 17.03 & 83.62 & 85.08 & 81.35 & 84.91 & 15.380 & -13.885 & 12.252 & -4.422 & 21.5 \\
\hline $5 e$ & 31.32 & 68.33 & 75.13 & 65.44 & 59.30 & 24.200 & -11.730 & 2.508 & -21.018 & 23.0 \\
\hline $6 a$ & 20.87 & 78.52 & 81.25 & 75.12 & 20.87 & 00.000 & 00.000 & 00.000 & 00.000 & 16.0 \\
\hline $6 b$ & 19.03 & 55.02 & 50.64 & 70.09 & 19.03 & 18.241 & 5.898 & -16.608 & -8.889 & 24.0 \\
\hline $6 c$ & 11.55 & 58.16 & 61.25 & 39.89 & 37.55 & 60.783 & 32.801 & -29.995 & -40.784 & 19.5 \\
\hline $6 d$ & 35.14 & 57.51 & 67.04 & 58.57 & 35.14 & 29.597 & -15.191 & -13.849 & -21.294 & 21.9 \\
\hline $6 e$ & 34.78 & 29.45 & 54.43 & 42.75 & 44.78 & 61.785 & -30.323 & -26.821 & -37.278 & 22.0 \\
\hline $6 f$ & 19.10 & 45.16 & 49.04 & 67.08 & 19.10 & 33.674 & 4.888 & -32.214 & -8.852 & 25.0 \\
\hline $6 g$ & 12.90 & 62.11 & 63.44 & 78.27 & 12.90 & 8.418 & 2.539 & 16.810 & 6.948 & 23.5 \\
\hline $6 \mathrm{~h}$ & 40.07 & 33.22 & 52.05 & 39.66 & 40.07 & 59.098 & -32.735 & -29.195 & -39.607 & 21.0 \\
\hline $6 i$ & 15.48 & 86.27 & 87.65 & 79.83 & 15.48 & 9.888 & 2.948 & 6.404 & 6.933 & 24.0 \\
\hline
\end{tabular}
color difference; $\Delta H^{*}$, hue difference; $\mathrm{K} / \mathrm{S}=$ Amount of dye absorbed on the surface of the fabrics.

Table 2. Fastness properties of monoazo disperse dyes $\mathbf{5 a - e}$ and $\mathbf{6 a - i}$ on polyester fabrics.

\begin{tabular}{|c|c|c|c|c|c|c|}
\hline \multirow[t]{2}{*}{ Dyes } & \multirow[t]{2}{*}{ Washing } & \multirow[t]{2}{*}{ Acidic perspiration } & \multicolumn{2}{|c|}{ Rubbing } & \multirow[t]{2}{*}{ Sublimation at $180^{\circ} \mathrm{C}$} & \multirow[t]{2}{*}{ Light $(40 \mathrm{~h})$} \\
\hline & & & Dry & Wet & & \\
\hline $5 a$ & 4 & $3-4$ & 4 & 4 & 4 & $6-7$ \\
\hline $5 b$ & 4 & $3-4$ & 4 & 4 & $3-4$ & $6-7$ \\
\hline $5 c$ & 4 & $3-4$ & 4 & 4 & 4 & $6-7$ \\
\hline $5 d$ & 4 & $3-4$ & 4 & 4 & $3-4$ & $6-7$ \\
\hline $5 e$ & 4 & $3-4$ & 4 & 4 & 4 & $6-7$ \\
\hline $6 a$ & 4 & 4 & 4 & 4 & 4 & 5 \\
\hline $6 b$ & 4 & 4 & $3-4$ & $3-4$ & 4 & $4-5$ \\
\hline $6 c$ & 4 & 4 & 4 & 4 & $4-5$ & $5-6$ \\
\hline $6 d$ & 4 & $3-4$ & 4 & 4 & 4 & $5-6$ \\
\hline $6 e$ & $4-5$ & $4-5$ & 4 & 4 & 4 & 6 \\
\hline $6 f$ & 4 & $3-4$ & $3-4$ & 4 & 4 & 5 \\
\hline $6 \mathrm{~g}$ & $4-5$ & $3-4$ & 4 & 4 & 4 & 6 \\
\hline $6 \mathrm{~h}$ & 4 & $4-5$ & 4 & 4 & 4 & 5 \\
\hline $6 i$ & $4-5$ & $3-4$ & $3-4$ & 4 & $4-5$ & $4-5$ \\
\hline
\end{tabular}

\subsubsection{Fastness to perspiration}

The samples were prepared by stitching a piece of dyed polyester fabric between two pieces of cotton and wool fabrics, all of equal length, and then immersed in the acid or alkaline solution for $30 \mathrm{~min}$. The staining on the undyed adjacent fabrics was assessed according tothe following gray scale: 1-poor, 2fair, 3-moderate, 4-good, 5-excellent. The acid solution $(\mathrm{pH}$ $=4.5)$ contains sodium chloride $(10 \mathrm{~g} / \mathrm{L})$, sodium dihydrogen orthophosphate $(1 \mathrm{~g} / \mathrm{L})$ and histidine monohydrochloride $(0.25$ $\mathrm{g} / \mathrm{L})$. The alkaline solution $(\mathrm{pH}=8.7)$ contains sodium chloride $(10 \mathrm{~g} / \mathrm{L})$, disodium orthophosphate $(1 \mathrm{~g} / \mathrm{L})$ and histidine monohydrochloride $(0.25 \mathrm{~g} / \mathrm{L})$.

\subsubsection{Fastness to light}

Light fastness was determined by exposing the dyed polyester on a Xenotest 150 (Original Hanau, chamber temperature: $25-30{ }^{\circ} \mathrm{C}$, black panel temperature: $60^{\circ} \mathrm{C}$, relative humidity: 50-60\%, dark glass UV filter system) for $40 \mathrm{~h}$. The changes in color were assessed according to the International Geometric blue scale: 1-poor, 3-moderate, 4-good, 6-very good, 8-excellent.

\subsubsection{Fastness to sublimation}

This test was made according to the ISO/R, 105/IV-1968 pt. 2. The dyed sample was sandwiched between two undyed samples (one from cotton and the other from the same fiber under test) and then placed in iron tester (Yasuda no. 138) at $180^{\circ} \mathrm{C}$ for 30 seconds. Change in color of the-dyed samples and staining of the-undyed ones were assessed using International Geometric Grey Scale (1-5; 1-poor, 2-fair, 3-moderate, 4-good, 5-excellent).

\subsubsection{Fastness to rubbing}

Two pieces of the dyed fabric (one dried and the other completely wetted with distilled water) were placed alternatively on the base of the Crockmeter, so that it rested flat on the abrasive cloth with its long dimension in the direction of rubbing. A square of white testing cloths were allowed to slide on the tested fabric back and forth twenty times by making ten complete turns of the crank according to the international standard procedures. The same procedures were applied to the wetted sample. The staining on the white testing cloth was assessed according to the International Geometric grey scale.

\section{Results and discussion}

3-Amino-4,5-diphenyl-1H-pyrazolo[3,4-c]pyridazine 2, was prepared in $85 \%$ yield, as yellow crystalline (M.p. $244-245^{\circ} \mathrm{C}$ ) according to the reported procedures [7] by refluxing 3-chloro5,6-diphenylpyridazine-4-carbonitrile 1 with hydrazine hydrate for 3 hours, (Scheme 1).

Compound 2, when reacted with ethyl azobenzene acetoacetate derivatives 3a-e in a mixture of ethanol and pyridine at refluxing temperature yielded 7-arylazo-6-methyl3,4-diphenylpyrimido[1',2:1,5]pyrazole[3, 4-c]pyridazin$8(5 \mathrm{H})$-one derivatives 5a-e (Scheme 2).

Compound 2, when reacted with the azobenzene malononitrile derivatives $\mathbf{4 a - I}$ in ethanol at refluxing temperature yielded 6,8-diamino-7-arylazo-3,4-diphenyl pyrimido [1',2:1,5]pyrazolo[3,4-c]pyridazine 6a-i (Scheme 3).

The 3-[4-(arylazo)-3,5-disubstituted-pyrazol-1-yl]-4,5-di phenyl-1H-pyraz-olo[3,4-c]pyridazines 5a-e and 6a-I were synthesized to assess their dyeing properties on polyester fabrics. The dyeing was performed at $2 \%$ shade by hightemperature techniques and gave generally deep and bright intense hues, ranging from yellow to orange-yellow.

The values of $\mathrm{K} / \mathrm{S}$ of compounds $\mathbf{5 a - e}$ and $\mathbf{6 a}-\mathbf{i}$ vary from 16 to 25 . The introduction of different groups in dyes $\mathbf{5 a - e}$ and 6a-i increases the strength of K/S values and deepens the color compared with the parent dyes $\mathbf{5 a}$ and $\mathbf{6 a}$, respectively (Table 1 ). The values of $K / S$ for the dyes $\mathbf{5 a}-\mathbf{c}, \mathbf{e}$ derived from ethyl azobenzene acetoacetate derivatives 3a-c,e with 3-amino pyrazolopyridazine $\mathbf{2}$ were greater than the corresponding 
dyes 6a-c, e derived from azobenzene malononitrile derivatives $\mathbf{4 a - c}$, e with the same 3-aminopyrazolopyridazine $\mathbf{2}$ on dyed polyester fibers. The color hues of the dyes 5a-e and6a-i on polyester fabrics are shifted towards the reddish and yellowish directions on the red-green and yellow-blue axes, respectively.

Most influences that can affect fastness are light, washing, heat, perspiration, and atmospheric pollution. Conditions of such tests are chosen to correspond closely to treatments employed in manufacture and ordinary use conditions [11]. Results are given after usual matching of tested samples against standard reference (the grey scale) [11-13]. The results revealed that these dyes have good-excellent fastness properties (Table 2).

\section{Conclusions}

A set of 14 disperse dyes 5a-e and 6a-i were synthesized by reaction of3-amino-4,5-diphenyl- $1 H$-pyrazolo[3,4-c]pyridazine 2 with ethyl arylazo acetoacetate and arylazo malononitrile derivatives. All of them were investigated for their dyeing characteristics on polyester. The dyed fabrics exhibit very good to excellent (4-5) washing, perspiration, rubbing and sublimation fastness properties. The remarkable degree of levelness and brightness after washings is indicative of good penetration and the excellent affinity of these dyes for the fabric due to the accumulation of polar groups. This in combination with the ease of preparation makes them particularly valuable.

\section{References}

[1]. Abdel Galil, F. M.; Khalifa, F. A.; Abdin, T. S. Dyes Pigments 1990, 12, 49-56.

[2]. Rajagopal, R.; Seshadri, S. Dyes Pigments 1990, 13, 93-105.

[3]. Deeb, A.; Shaqra, S.; Abo El-Fotoh, T.; Dief, S. Eur. Chem. Bull. 2014, 3(7), 627-636.

[4]. Deeb, A.; El-Hossami, M.; Awad A. Eur. Chem. Bull. 2014, 3(8), 745751.

[5]. Deeb, A.; Yassine, F.; Aouf, N.; Shehta, W. Int. J. Chem Tech Res. 2014, 6(1), 719-729.

[6]. Deeb, A.; Yassine, F.; Aouf, N.; Shehta, W. Eur. Chem. Bull. 2014, 3(1), 18-23.

[7]. Deeb, A.; Bayoumy, B.; Essawy, A.; Fikry, R. Heterocycles 1991, 32, 895-900.

[8]. Deeb, A.; Bayoumy, B.; Essawy, A.; Fikry, R. Heterocycles 1991, 32, 901-907.

[9]. Deeb, A.; Yassin, F.; Ouf, N.; Shehta, W. Chem. Heterocycl. Compd. 2010, 46, 212-222.

[10]. Shawali, A. S.; Abdel Galil, F. M. Tetrahedron 1971, 27, 4305-4316.

[11]. Chrysler, L. P. Methods of Test for Color Fastness of Textiles and Leather, $7^{\text {th }}$ ed., British Standards Institution, London, Bradford, 1990, pp. 89-94.

[12]. Al-Etaibi, A.; El-Apasery, M. A.; Al-Awadi, N. Eur. J. Chem. 2013, 4(3), 240-244.

[13]. Al-Qalaf, F.; Almohammad, K.; El-Apasery, M. A.; Mohmoud, H. Eur. J. Chem. 2013, 4(3), 211-215. 